\title{
Association of Maternal Histocompatibility at Class II HLA Loci with Maternal Microchimerism in the Fetus
}

\author{
STANLEY M. BERRY, SONIA S. HASSAN, EVELYNE RUSSELL, DEBRA KUKURUGA, \\ SUSAN LAND, AND JOSEPH KAPLAN
}

Division of Maternal-Fetal Medicine [S.M.B., S.S.H., E.R.], Department of Obstetrics and Gynecology, Departments Pathology [D.D.] and Pediatrics [J.K.], and Center for Molecular Medicine and Genetics

[S.L.], Wayne State University, Detroit, MI 48201, U.S.A.

\begin{abstract}
For investigating the possible influence of maternal-fetal HLA compatibility on maternal microchimerism, DNA samples from blood of 120 maternal-fetal pairs were genotyped at two polymorphic loci: glutathione-S-transferase M1 (GSTM1) and angiotensin-converting enzyme $(A C E)$. Informative pairs (mother heterozygous/fetus homozygous at one of the two loci) were then tested by quantitative real-time PCR for the noninherited maternal allele(s) and genotyped at the HLA-A, B, and C class I loci and/or at the DRB1 and/or DQB1 class II loci. Small numbers of maternal cells were detected in the circulation of 16 of the 30 informative second- and third-trimester fetuses. Comparison with HLA data suggested an association between micro-
\end{abstract}

ABSTRACT

chimerism and maternal compatibility at the class II DRB1 and/or DQB1 HLA loci and with the maternal HLA-DQB1*0301 allele. There was no relationship between maternal microchimerism and maternal-fetal HLA compatibility at other HLA loci or with gestational age, fetal anomalies, or red cell or platelet isoimmunity. (Pediatr Res 56: 73-78, 2004)

\section{KIR, killer inhibitory receptor}

NIMA, non-inherited maternal allele

NK cell, natural killer cell

QPCR, quantitative PCR
Transplacental passage of small numbers of maternal and fetal nucleated cells, including hematopoietic stem cells, is a common occurrence that often results in persistent fetal or maternal microchimerism - the presence of small numbers of fetal or maternal cells in mothers or their progeny, respectively (1-3). This raises the possibility that maternal and fetal microchimerism may play a role in such clinically important phenomena as immune ontogeny, vertical transmission of infections, and tissue repair and regeneration by transdifferentiated stem cells. Particular interest has focused on the possibility that chimeric maternal and fetal alloreactive lymphocytes may play a role in autoimmunity (4-6). Although an increasing number of clinical studies have addressed this question, there has been a paucity of information concerning the factors that regulate the presence and levels of naturally occurring maternal and fetal chimeric cells.

Among the possible regulatory factors that might be involved, maternal-fetal histocompatibility seems to be a prime

Received June 19, 2003; accepted February 6, 2004.

Correspondence: Joseph Kaplan, M.D., Children's Hospital of Michigan, 3901 Beaubien Boulevard, Detroit, MI 48201, U.S.A.; e-mail: jkaplan@med.wayne.edu

Supported by grants from the Department of Obstetrics and Gynecology of Wayne State University School of Medicine, and the Children's Research Center of Michigan.

DOI: 10.1203/01.PDR.0000129656.10005.A6 candidate in view of the importance of histocompatibility in regulating hematopoietic chimerism in both adult and fetal recipients of adult bone marrow. In this study, we directly tested for the first time the influence of maternal-fetal histocompatibility and specific HLA alleles on both the presence and level of maternal microchimerism in the fetus. The results strongly suggest that maternal microchimerism in fetal blood is associated with maternal compatibility at the class II DRB1 and/or DQB1 HLA loci and is likely also associated with the maternal HLA-DQB $1 * 0301$ allele.

\section{METHODS}

Study subjects. Paired whole-blood samples were obtained from 120 maternal-fetal pairs who underwent fetal blood sampling for a variety of medical indications, including red cell or platelet alloimmunization, and the need for rapid karyotyping mandated by an ultrasound diagnosis of fetal anomalies. The gestational age at fetal blood sampling ranged from $18.3 \mathrm{wk}$ to 38 wk (mean, $27.2 \mathrm{wk}$; median, $26.5 \mathrm{wk}$ ). We excluded from the study fetuses who had received intrauterine blood or platelet transfusions before the cordocentesis procedure and fetuses with placental abruption. Maternal consent was obtained for storage of the excess specimens for research purposes under a 
protocol approved by the Wayne State University Institutional Review Board. The current study was separately approved by the same committee. Cordocentesis and venipuncture were used to obtain 1 to $6 \mathrm{~mL}$ of fetal blood and 1 to $10 \mathrm{~mL}$ of maternal blood, respectively. The method of cordocentesis has been previously described (7).

Preparation of genomic DNA. Genomic DNA was prepared from each whole-blood specimen using the QIAamp DNA Extraction Kit for PCR (QIAGEN, Mississauga, Ontario, Canada) according to the manufacturer's directions. All DNA samples were stored frozen at $-70^{\circ} \mathrm{C}$.

Real-time quantitative PCR assays for chimeric maternal cells. We took advantage of two different polymorphic locithe deletion polymorphism of the glutathione S-transferase M1 (GSTM1) gene and the insertion/deletion polymorphisms of intron 16 of the angiotensin-converting enzyme $(A C E)$ gene - to apply three different quantitative PCR (QPCR) assays for detection and quantification of noninherited maternal alleles (NIMAs) as markers of maternal cells in fetal blood. Informative maternal-fetal pairs are those in which the mother possesses an allele not present in the fetus. The GSTM1 locus is deleted in roughly half of the population, which permits detection of NIMA when the mother is GSTM1 positive and the fetus is GSTM1 negative. Genotyping of GSTM1 was performed as previously described by Lo et al. $(8,9)$ using primers GSTM1E4 (CTGCCCTACTTGATTGATGGG) and GSTM1E5 (CTGGATTGTAGCA GATCATGC) in 30 cycles of PCR $\left(94^{\circ} \mathrm{C} 1 \mathrm{~min}, 59^{\circ} \mathrm{C} 1 \mathrm{~min}\right.$, and $72^{\circ} \mathrm{C}$ for $1 \mathrm{~min} ; \mathrm{Mg}^{2+}$ concentration $1.5 \mathrm{mM}$ ). Amplification of DNA from GSTM1positive individuals produces a 273 -bp product. $A C E$ polymorphism is characterized by insertion or deletion of a $287-\mathrm{bp}$ fragment in intron 16 of the $A C E$ gene. NIMA can be detected when the mother is heterozygous for the insertion and deletion alleles and the fetus is homozygous for either allele. The insertion and deletion alleles yield 479-bp and 191-bp products, respectively. Genotyping of $A C E$ was performed using primers ACE-P1 (CTGGAGACCACTCCCATCCTTTCT) and ACE-P2 (GATGTGGCCATCACATTCGTCAGA) using 30 cycles of PCR $\left(94^{\circ} \mathrm{C} 1 \mathrm{~min}, 58^{\circ} \mathrm{C} 1 \mathrm{~min}\right.$, and $72^{\circ} \mathrm{C} 1 \mathrm{~min}$; $\mathrm{Mg}^{2+}$ concentration of $3 \mathrm{mM}$ ).

The Taqman QPCR assay used in these studies is based on continuous monitoring of the accumulation of a fluorescent product released during each PCR cycle of a PCR amplification reaction when the $5^{\prime}$ to $3^{\prime}$ exonuclease activity of Taq polymerase cleaves it from an oligonucleotide probe hybridizing to the PCR product. In the experiments reported here, the fluorescent signals were detected in real time using an ABI Biosystems 7700 Sequence Detector (ABI Biosystems, Foster City, CA 94404).

For detecting and quantifying NIMAs in fetal DNA samples, four QPCR assay systems were used exactly as described by Lo et al. (9): one for the GSTM1 gene, one for the $A C E$ insertion allele, one for the $A C E$ deletion allele, and one for the $\beta$-globin gene. The last was used in combination with each of the other assays as a positive template control for each QPCR reaction and to calculate the fractional concentration of the noninherited allele. A normal diploid cell was defined as possessing one genome equivalent of the $\beta$-globin sequence. Each assay was carried out in duplicate using $500 \mathrm{ng}$ of template per reaction with the mean results used for further analysis. For controlling for possible inhibition of QPCR amplification reactions by excess template, reactions were also carried out with $250 \mathrm{ng}$ of template, and in almost all instances, the results obtained were identical at the two different template doses.

In $A C E$ assays for the insertion (I) or deletion (D) alleles, a standard curve was constructed using as a positive control genomic DNA from a human subject who was known to be heterozygous ID. Negative control DNA was derived from established cell lines that are known to lack either the insertion allele (cell line Detroit 1564; Cell Culture Laboratory, Detroit, MI, U.S.A.) or the deletion allele (cell line CEPH; ABI Biosystems). In the GSTM1 assay, the positive and negative controls were human genomic DNA samples that were known to be either GSTM1 positive or GSTM1 null. In each assay, two standard curves were constructed: one standard curve for the test allele and one standard curve for the universally present $\beta$-globin gene as a reference standard for the amount of genomic DNA in the test template. For purposes of calculating fractional concentrations of NIMAs on the basis of the standard curves, a single cell was considered to contain 6.6 pg of DNA equal to one genome equivalent of the respective allele. Thus, the fractional concentration of NIMAs present in each 500-ng sample of fetal genomic DNA used as template was calculated as genome equivalents of NIMA/genome equivalents $\beta$-globin. Each sample of $500 \mathrm{ng}$ of genomic DNA contained $\sim 83,000$ genome equivalents of $\beta$-globin, and on the basis of their standard curves, the $\beta$-globin, GSTM1, and ACE systems each were able to detect as few as one to three genome equivalents. However, direct comparison of the three QPCR assays showed that the QPCR assay for the $A C E$ insertion allele was consistently less sensitive than the assays for either GSTM1 or $A C E$ deletion polymorphisms. For this reason, we included in our analysis only maternal-fetal pairs informative at either the GSTM1 or ACE deletion polymorphisms. A representative standard curve for the GSTM1 allele is shown in Figure 1.

HLA genotyping. NIMA-informative maternal-fetal pairs were genotyped for alleles at the HLA-A, B, C, DRB1, and

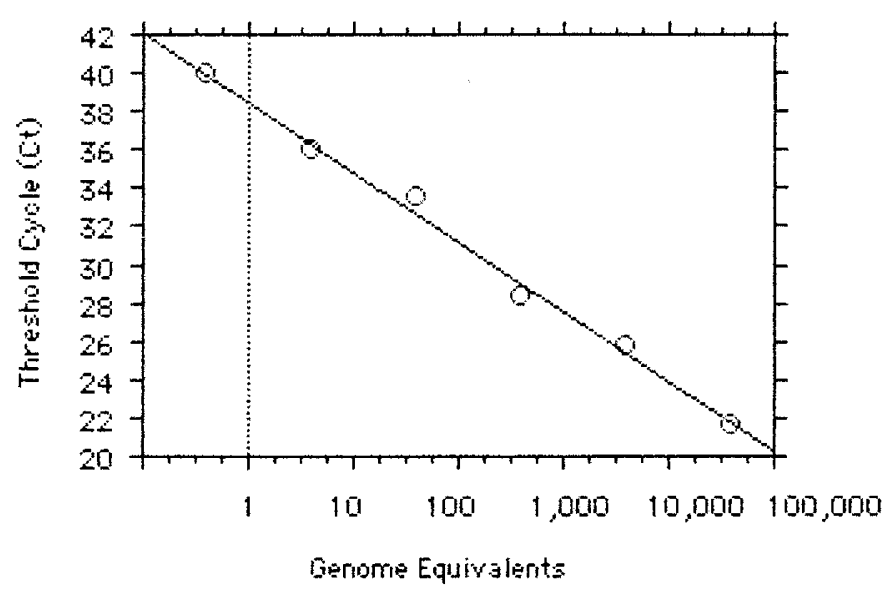

Figure 1. Real-time QPCR analysis of the GSTM1 gene. Plot of the threshold cycle (Ct, cycle at which signal above background is first detected) against the input target quantity (logarithmic scale). 
DQB1 loci by intermediate resolution DNA-based typing using PCR amplification and reverse sequence-specific oligonucleotide probe kits (Dynal RELI SSO HLA Tests; Dynal Biotech, Oslo, Norway). High-resolution typing for DRB1 or DQB1 alleles was initially performed by DNA sequencing in those cases in which results of intermediate resolution typing were ambiguous. Additional samples underwent allele-specific highresolution typing of the DQB1*03xx and DQB1*06xx allele families.

Statistical analysis. Odds ratios were calculated as the ratios of the proportions of maternal-fetal pairs with a given pattern of maternal-fetal compatibility with detectable chimeric maternal cells to the proportion of those pairs without detectable chimeric maternal cells. On the basis of their HLA genotypes, we assigned each mother or fetus as "compatible" or "incompatible" from the perspective of the alloreactive $\mathrm{T}$ cells or natural killer (NK) cells of the opposite member of the pair. Maternal compatibility from the perspective of fetal alloreactive $\mathrm{T}$ cells was considered to exist when none of the maternal alleles differed from those detected in the fetus. Similarly, fetal compatibility from the perspective of the mother's alloreactive $\mathrm{T}$ cells was considered to exist when none of the fetal alleles differed from those detected in the mother. Using the algorithm described by Ruggeri et al. (10), maternal compatibility from the perspective of fetal NK cells was considered to exist when the mother lacked none of the class I allele group(s) recognized by killer inhibitory receptors (KIRs) of her fetus, and fetal compatibility from the perspective of maternal NK cells was considered to exist when the fetus lacked none of the class I allele group(s) recognized by KIRs of its mother. (The currently identified class I allele KIR ligand-defining groups are HLA-Cw2,4,5,6, and related alleles; HLA-Cw1, 3,7,8 and related alleles; HLA-Bw4 alleles; and HLA-A3/A11.)

Exact methods were used to calculate $95 \%$ confidence intervals and two-sided $p$ values associated with the ratios. Between-group differences in levels of maternal microchimerism were compared by the Mann-Whitney log-rank test. Statistical significance was set at $p \leq 0.05$.

\section{RESULTS}

Prevalence and levels of maternal microchimerism in fetal blood. Genotyping of 120 maternal-fetal DNA sample pairs at the GSTM1 and ACE loci revealed that 30 fetal samples were informative for detection of maternal microchimerism by QPCR detection of the noninherited maternal GSTM1 or ACE deletion allele 21 at the GSTM1 locus (mother GSTM1 positive/fetus GSTM1 null) and 9 at the $A C E$ locus (mother heterozygous for the $A C E$ insertion and deletion alleles, and fetus homozygous for the $A C E$ insertion allele).

The results of testing the 30 informative maternal-fetal pairs for maternal microchimerism are shown together with selected

Table 1. Presence and levels of maternal cells in fetal blood

\begin{tabular}{|c|c|c|c|c|}
\hline $\begin{array}{l}\text { Maternal-fetal } \\
\text { pair }\end{array}$ & $\begin{array}{c}\text { Fraction of maternal } \\
\text { cells } \times 10^{-5}\end{array}$ & $\begin{array}{l}\text { Gestational } \\
\text { age (weeks) }\end{array}$ & Cordocentesis indication & $\begin{array}{c}\text { Transplacental } \\
\text { cordocentesis } \\
(\text { yes/no) }\end{array}$ \\
\hline 7 & $<1$ & 29 & Rapid karyotype & No \\
\hline 9 & $<1$ & 22.3 & Rh alloimmunization & No \\
\hline 10 & $<1$ & 35.2 & Rapid karyotype & No \\
\hline 19 & 8 & 20.5 & Kell alloimmunization* & No \\
\hline 22 & $<1$ & 38 & Fetal platelet count* & No \\
\hline 29 & $<1$ & 24.2 & $\mathrm{Rh}$ alloimmunization & No \\
\hline 32 & 1 & 30.1 & Kell alloimmunization* & No \\
\hline 39 & 2 & 27.5 & Rapid karyotype & Yes \\
\hline 52 & 30 & 26.3 & Rapid karyotype & No \\
\hline 63 & $<1$ & 34.6 & Rapid karyotype & No \\
\hline 65 & 4 & 19.6 & ? Fetal infection & No \\
\hline 66 & 89 & 24.5 & Intrauterine growth retardation & No \\
\hline 69 & 10 & 22.2 & $\mathrm{Rh}$ alloimmunization & Yes \\
\hline 72 & $<1$ & 18.3 & Rapid karyotype & No \\
\hline 73 & 10 & 25 & Rapid karyotype & No \\
\hline 80 & 3 & 29.6 & $\mathrm{Rh}$ alloimmunization & Yes \\
\hline 109 & 50 & 22 & Fetal platelet count & No \\
\hline 112 & $<1$ & 32.6 & ? Fetal infection & No \\
\hline $2-7$ & $<1$ & 22 & Oligohydramnios & No \\
\hline $2-8$ & $<1$ & 30.2 & Polyhydramnios & Yes \\
\hline $2-9$ & 37 & 33.5 & Oligohydramnios & Yes \\
\hline $2-11$ & 687 & 26 & $\mathrm{Rh}$ alloimmunization & Yes \\
\hline $2-12$ & 80 & 30 & $\mathrm{Rh}$ alloimmunization & Yes \\
\hline $2-13$ & $<1$ & 26 & $\mathrm{Rh}$ alloimmunization & No \\
\hline $2-14$ & $<1$ & 24 & Fetal platelet count & Yes \\
\hline $2-16$ & 160 & 26 & $\mathrm{Rh}$ alloimmunization & Yes \\
\hline $2-17$ & 83 & 32.3 & $\mathrm{Rh}$ alloimmunization & Yes \\
\hline $2-18$ & $<1$ & 32.4 & Rh alloimmunization & No \\
\hline $2-19$ & $<1$ & 26.6 & $\mathrm{Rh}$ alloimmunization & Yes \\
\hline $2-25$ & 316 & 26.3 & $\mathrm{Rh}$ alloimmunization & No \\
\hline
\end{tabular}

* Test revealed no abnormality. 
clinical parameters in Table 1. Maternal genomic DNA was detectable by QPCR in the blood of 16 of the 30 informative fetuses. No attempt was made to separate cells from plasma before whole-blood DNA extraction. However, because $\sim 99 \%$ of DNA in whole blood is associated with intact cells (9), the fraction of maternal DNA detected in these assays almost entirely reflects the relative fraction of maternal cells to fetal cells. The fractional concentration of maternal cells in fetal blood ranged from $1 \times 10^{-5}$ to $687 \times 10^{-5}$, with a median fractional concentration of $13 \times 10^{-5}$ and a mean fractional concentration of $89 \times 10^{-5}$. Odds ratio analysis and nonparametric log-rank tests revealed no statistically significant relationships between the presence or level of maternal microchimerism and average gestational age at blood sampling, maternal gravidity, maternal parity, maternal age, or use of a transplacental as opposed to a transamniotic echo-visualized approach to cordocentesis. (This last finding is evidence that the blood sampling procedure did not enhance maternal cell passage into the fetal circulation.) We also observed no association between the presence or levels of detectable maternal cells and the indication for cordocentesis including the presence of fetal anatomical anomalies, infection, or antibodyinduced abnormalities of red cells or platelets. One of the fetuses who had been karyotyped because of a possible anatomical anomaly (7) and five of the fetuses who had been considered potential targets for maternal alloimmunization $(9$, $19,22,32$, and 109) subsequently turned out to be "normal" by virtue of a normal karyotype and normal anatomy at birth, absence of detectable maternal antibody to fetal erythrocytes, or normal platelet count. The proportion of these "normal" fetuses with chimeric maternal cells ( 3 of 6 ) was similar to the proportion of "abnormal" fetuses with such cells (13 of 24).

Influence of maternal-fetal HLA compatibility on the presence and levels of maternal microchimerism. For examining the possible relationship between maternal-fetal histocompatibility and maternal microchimerism, maternal-fetal pairs that had been tested by QPCR for maternal microchimerism were genotyped at three HLA class I loci-HLA-A, B, C-and two HLA class II loci-DRB1 and DQB1. After HLA genotyping had been completed on 18 maternal-fetal pairs, preliminary analysis of the results indicated that histocompatibility at any of the class I HLA-A, B, and C loci had little if any apparent influence on either the presence or level of maternal microchimerism, but it raised the possibility of an association with maternal-fetal compatibility at the class II HLA-DRB1 and DQB1 loci. With that in mind, we limited HLA genotyping to the DRB1 and DQB1 class II loci on the remaining 10 informative maternal-fetal pairs for which sufficient DNA was available. Because the resulting cumulative data pointed to the possible importance of maternal-fetal histocompatibility at the DQB1*03xx and DQB1*06xx families of alleles, we subsequently carried out further high-resolution genotyping for specific DQB1*03xx and DQB1*06xx alleles on all available DNA samples from each of the 30 maternal-fetal pairs that possessed one or more of those particular alleles. Table 2 presents the final tabulation of the completed HLA typing data on all maternal-fetal pairs so tested together with their levels of maternal microchimerism.
Analysis of this complete data set (Table 3) revealed a positive association between maternal compatibility from the fetal perspective at HLA-DQB1 and DRB1 and the presence of maternal microchimerism in the fetal circulation. The data showed that 5 of the 28 tested fetuses had HLA-DQB1compatible mothers, and in 2 of these 5 fetuses, the mothers were also HLA-DRB1 compatible. All five of the fetuses with DQB1-compatible mothers including the two with DRB1compatible mothers contained chimeric maternal cells. By contrast, only 9 of the 23 fetuses with DQB1 and DRB1incompatible mothers contained such cells (odds ratio, infinity; $p=0.04$ ). This strongly suggests that maternal histocompatibility at HLA-DRB1 and/or HLA-DQB1 favors but is not required for the occurrence of maternal microchimerism in the fetus. Fetuses with DQB1-compatible mothers also tended to have higher mean fractional levels of maternal microchimerism $(8.8 \times 10 \mathrm{E}-4)$ than the latter group $(1.3 \times 10 \mathrm{E}-4 ; p=0.09$ by the Mann-Whitney test.) By contrast, fetal histocompatibility at either DBQ1 or DRB1 from the perspective of the mother showed no relationship to either the presence or level of maternal microchimerism.

We then analyzed the data for possible associations between specific DQB1*03xx and DQB1*06xx alleles and maternal microchimerism. This revealed a likely association between the presence of maternal microchimerism in the fetus and maternal DQB $1 * 0301$, a weaker possible association with fetal DQB ${ }^{*} 0301$, and an intermediate strength association with combined maternal and fetal DQB1*0301. By contrast, as illustrated by the results of analysis of another common DQB1 allele, DQB1*0602, we detected no apparent associations between other maternal or fetal class II alleles and the presence or levels of maternal microchimerism in the fetal blood.

\section{DISCUSSION}

Our results strongly suggest that maternal microchimerism in the fetal circulation is associated with maternal compatibility from the fetal perspective at HLA-DQB1 and, possibly, at HLA-DRB1. These findings are consistent with previous data suggesting that class II compatibility may influence donor cell chimerism. Thus, Tajik et al. (11) previously documented an association between the degree of donor class II HLA compatibility from the recipient's perspective and post-organ transplant donor cell microchimerism, and Nelson et al. (12) reported that women with scleroderma have a higher level of male cell (presumably fetal) microchimerism than control women and are more likely to have class II HLA-compatible sons than class II HLA-incompatible sons. Taken together, these findings suggest that absence of fetal alloimmune recognition of maternal class II HLA antigens favors maternal microchimerism.

Our data further suggest that maternal microchimerism is favored by the presence of maternal DQB1*O301 and, possibly, sharing of $\mathrm{DQB} 1 * 0301$ by both mother and fetus. This fits with previous findings indicating that $\mathrm{DQB} 1 * 0301$ is associated with scleroderma (13), a condition associated with microchimerism, as described above. How the presence of maternal DQB1*0301 might influence maternal microchimerism is a 


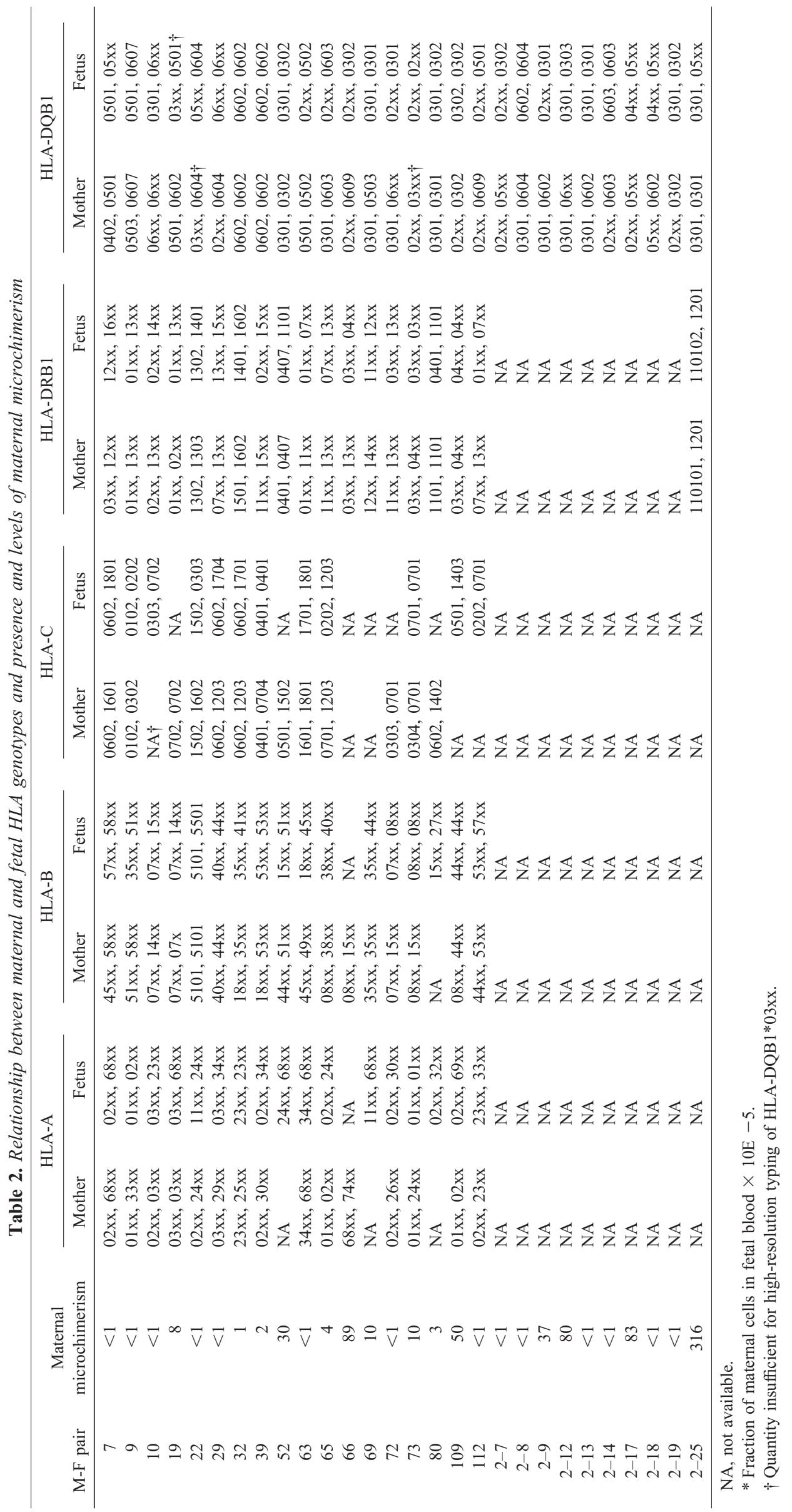


Table 3. Influence of HLA-class II histocompatibility and DQB1 alleles on maternal microchimerism

\begin{tabular}{|c|c|c|c|c|}
\hline & $\begin{array}{l}\text { Fraction of tested } \\
\text { fetuses with } \\
\text { maternal cells }\end{array}$ & $\begin{array}{c}\text { Fraction of tested } \\
\text { fetuses without } \\
\text { maternal cells }\end{array}$ & $\begin{array}{c}\text { Odds ratio } \\
(95 \% \mathrm{CI})\end{array}$ & $\begin{array}{l}\text { Fisher } \\
\text { exact } p\end{array}$ \\
\hline \multicolumn{5}{|l|}{ HLA class II histocompatibility } \\
\hline Fetal DQB $1 \dagger$ & $5 / 13$ & $2 / 11$ & $2.8(0.3-29)$ & 0.39 \\
\hline Maternal DRB1* & $2 / 12$ & $0 / 6$ & $\operatorname{Inf}(0.1-$ inf $)$ & 0.53 \\
\hline \multicolumn{5}{|l|}{ DQB1 allele } \\
\hline Maternal and fetal DQB $1 * 0301$ & $6 / 14$ & $1 / 14$ & $9.8(0.8-258)$ & 0.08 \\
\hline Maternal DQB $1 * 0602$ & $5 / 13$ & $2 / 14$ & $3.8(0.4-38)$ & 0.21 \\
\hline Fetal DQB $1 * 0602$ & $2 / 14$ & $1 / 11$ & $1.3(0.1-59)$ & $>0.99$ \\
\hline
\end{tabular}

CI, confidence interval.

* Histocompatible from the perspective of the fetus.

$\uparrow$ Histocompatible from the perspective of the mother.

matter for speculation. One testable possibility is that fetal recognition of this particular allele on maternal cells somehow results in primary stimulation of $\mathrm{T}$ cells with suppressor activity for $\mathrm{T}$ cell reactivity to other maternal alloantigens.

We made no attempt in this study to determine the nature of the maternal cells found in fetal blood or whether they are retained until birth and beyond. However, others have shown that chimeric maternal cells in newborn cord blood include CD34 progenitor cells (14) as well as mature polymorphonuclear cells (15). Thus, it seems that chimeric maternal cells comprise, at the very least, maternal hematopoietic stem cells and their progeny. This conclusion, together with growing evidence that at least some transplanted adult multipotent bone marrow stem cells can differentiate into mature nonhematopoietic cells, particularly in injured tissues $(16,17)$, raises the intriguing possibility that before and after birth, chimeric maternal stem cells could play a beneficial physiologic role in tissue repair and regeneration. Further efforts to address these and other potentially clinically relevant effects of maternal microchimerism would be greatly facilitated by more detailed understanding of the mechanisms of in vivo regulation of this phenomenon. The influence of maternal class II HLA compatibility on maternal microchimerism demonstrated here strongly suggests that one important regulatory mechanism involves the fetal immune system's interaction with maternal class II HLA antigens. We are currently using a mouse model of maternal microchimerism to test this hypothesis more directly.

Acknowledgments. We thank Drs. Shan-Rong Chou and Zhan Zhang for carrying out initial genotyping assays, Drs. Ronald Thomas and Xiao-Ming Li for help with statistical analyses, and Janelle Novak and Michelle Phillips for carrying out QPCR assays. All quantitative PCR experiments were carried out in the Wayne State University Genomics Facility.

\section{REFERENCES}

1. Bianchi DW, Zickwolf GK, Weil GJ, Sylvester S, DeMaria MA 1996 Male fetal progenitor cells persist in maternal blood for as long as 27 years postpartum. Proc Natl Acad Sci U S A 93:705-708

2. Evans PC, Lambert N, Maloney S, Furst DE, Moore JM, Nelson JL 1999 Long-term fetal microchimerism in peripheral blood mononuclear cell subsets in healthy women and women with scleroderma. Blood 93:2033-2037

3. Maloney S, Smith A, Furst DE, Myerson D, Rupert K, Evans PC, Nelson JL 1999 Microchimerism of maternal origin persists into adult life. J Clin Invest 104:41-47

4. Nelson JL 1998 Microchimerism and autoimmune disease. N Engl J Med 338:12241225

5. Reed AM, Picornell YJ, Harwood A, Kredich DW 2000 Chimerism in children with juvenile dermatomyositis. Lancet 356:2156-2157

6. Artlett CM, Ramos R, Jiminez SA, Patterson K, Miller FW, Rider LG 2000 Chimeric cells of maternal origin in juvenile idiopathic inflammatory myopathies. Childhood Myositis Heterogeneity Collaborative Group. Lancet 356:2155-2156

7. Berry SM, Fine N, Bichalski JA, Cotton DB, Dombrowski MP, Kaplan J 1992 Circulating lymphocyte subsets in second- and third-trimester fetuses: comparison with newborns and adults. Am J Obstet Gynecol 167:895-900

8. Lo YM, Lo ES, Watson N, Noakes L, Sargent IL, Thilaganathan B, Wainscoat JS 1996 Two-way cell traffic between mother and fetus: biologic and clinical implications. Blood 88:4390-4395

9. Lo YM, Lau TK, Chan LY, Leung TN, Chang AM 2000 Quantitative analysis of the bidirectional fetomaternal transfer of nucleated cells and plasma DNA. Clin Chem 46:1301-1309

10. Ruggeri L, Capanni M, Urbani E, Perruccio K, Shlomchik WD, Tosti A, Posati S, Rogaia D, Frassoni F, Aversa F, Martelli MF, Velardi A 2002 Effectiveness of donor natural killer cell alloreactivity in mismatched hematopoietic transplants. Science 295:2097-2100

11. Tajik N, Singal D, Pourmand G, Ebrahimi-Rad M, Radjabzadeh M, Tavasoli P, Khosravi F, Nikbin B 2001 Prospective study of microchimerism in renal allograft recipients: association between HLA-DR matching, microchimerism and acute rejection. Clin Transplant 15:192-198

12. Nelson JL, Furst DE, Maloney S, Gooley T, Evans PC, Smith A, Bean MA, Ober C, Bianchi DW 1998 Microchimerism and HLA-compatible relationships of pregnancy in scleroderma. Lancet 351:559-562

13. Tan FK, Stivers DN, Arnett FC, Chakraborty R, Howard R, Reveille JD 1999 HLA haplotypes and microsatellite polymorphisms in and around the major histocompatibility complex region in a Native American population with a high prevalence of scleroderma (systemic sclerosis). Tissue Antigens 53:74-80

14. Hall JM, Lingenfelter P, Adams SL, Lasser D, Hansen JA, Bean MA 1995 Detection of maternal cells in human umbilical cord blood using fluorescence in situ hybridization. Blood 86:2829-2832

15. Socie G, Gluckman E, Carosella E, Brossard Y, Lafon C, Brison O 1994 Search for maternal cells in human umbilical cord blood by polymerase chain reaction amplification of two minisatellite sequences. Blood 83:340-344

16. Theise ND, Nimmakayalu M, Gardner R, Illei PB, Morgan G, Teperman L, Henegariu O, Krause DS 2000 Liver from bone marrow in humans. Hepatology 32:11-16

17. Orlic D, Kajstura J, Chimenti S, Limana F, Jakoniuk I, Quaini F, Nadal-Ginard B, Bodine DM, Leri A, Anversa P 2001 Mobilized bone marrow cells repair the infarcted heart, improving function and survival. Proc Natl Acad Sci U S A 98:10344-10349 